COMMENT. The American Heart Association, in a most definitive statement and review of stroke in infants and children (Roach ES, et al. Stroke 2008;39:2644-2691), finds 55\% of strokes in children are ischemic and the remainder is hemorrhagic, whereas among adults, $85 \%$ are ischemic. About one-half of children presenting with an acute focal neurological deficit due to stroke have a previously identified risk factor, and the remainder has $>1$ additional risk factor. (Ganesan V, et al. Ann Neurol 2003;53:167173). The most common underlying conditions are SCD and congenital or acquired heart disease. Head trauma, often minor and sports related, is a trigger for arterial stroke (Kieslich M, et al. J Neurol Neurosurg Psychiatry 2002;73:13-16) and dehydration for venous stroke (deVeber G, et al. N Engl J Med 2001;345:417-423). Infections, including varicella, meningitis, tonsillitis, and otitis media are risk factors for both. Pediatric endocarditis was a risk factor for stroke in 7 patients reported from Children's Memorial Hospital, Chicago, 3 patients having mycotic aneurysms. (Venkatesan C, Wainwright MS. Pediatr Neurol 2008;38(4):243-247).

The peak age for ischemic stroke and intracerebral hemorrhage in children is in the first year of life, and one third of childhood stroke cases present in this age group. Subarachnoid hemorrhage is more common among teenagers. (Fullerton HJ, et al. Neurology 2003;61:189-194). An excess of strokes in boys and in those of African American ethnicity is independent of SCD.

\title{
NEONATAL ARTERIAL ISCHEMIC STROKE STUDY
}

The International Pediatric Stroke Study, a global research initiative of 149 coinvestigators ( 30 centers in 10 countries), examined predictors of infarct characteristics and outcome in patients with clinical and neuroimaging confirmation of symptomatic arterial ischemic stroke (AIS). Of 248 neonates enrolled 2003-2007, 57\% were male and $10 \%$ premature. Seizure was the presenting feature in $72 \%$ and nonfocal neurologic signs in $63 \%$. Vascular imaging infarcts on MRI, involving the anterior circulation and left hemisphere preferentially, were multifocal in 30\%. Maternal health and pregnancies were usually normal. Neonates required resuscitation in $30 \%$ and systemic illnesses were associated in 23\%. Cardiac and prothrombotic abnormalities were found in $<20 \%$, and antithrombotic treatment was used in only $21 \%$. Short-term outcome at discharge was poor, with deficits in 49\%. Long-term outcomes are pending. (Kirton A, ArmstrongWells J, Chang T et al. Symptomatic neonatal arterial ischemic stroke: The International Pediatric Stroke Study. Pediatrics Dec 2011;128(6):e1402-e1410). (Respond: Adam Kirton MD, Alberta Children's Hospital, 2888 Shaganappi Trail NW, Calgary, Alberta, Canada T3B 6A8. E-mail: adam.kirton@albertahealthservices.ca).

COMMENT. Neonates with arterial ischemic stroke (AIS) are often systemically ill, whereas their mothers are usually well. Risk factors for perinatal stroke include cardiac disorders, coagulation disorders, infection, trauma, drugs, maternal and placental disorders, and perinatal asphyxia. (Nelson KB, et al. Lancet Neurol 2004;3:150-158). The risk of perinatal arterial ischemic stroke increases dramatically with multiple risk factors. Long-term disabilities are frequent, and include cerebral palsy and cognitive impairments. Estimates of the incidence of cerebral palsy after AIS vary widely from $9 \%$ to $88 \%$. Although many neonates with AIS present with seizures, most do not develop 
epilepsy. (Roach ES, et al. Stroke 2008;39: 2644-2691). After perinatal brain injury, children tend to "grow into their defects," and the diagnosis of cerebral palsy is often delayed until 18 to 24 months; learning and behavior impairments are not appreciated until school age. (Ricci D et al. Stroke 2008;39(2):403-410).

\section{SEIZURE DISORDERS}

\section{GENETIC CLASSIFICATION OF INFANTILE SPASMS}

Researchers from University of Washington, Seattle, WA, and Washington University, St Louis, MO propose a genetic and biologic classification of infantile spasms. Infantile spasms are of 2 main groups: those with known or unknown predisposing genotypes. Genes associated with lissencephaly are strongly associated with infantile spasms, as high as $80 \%$, whereas $40 \%$ of patients with tuberous sclerosis complex may manifest infantile spasms. Inborn errors of metabolism associated with infantile spasms include the aminoacidopathies phenylketonuria, and nonketotic hyperglycinemia, the organic acidemias methylmalonic acidemia, propionic acidemia, and maple syrup urine disease, and Menkes kinky hair disorder of copper metabolism. Mitochondrial disorders are infrequently associated with infantile spasms.

Five chromosomal syndromes comprise predisposing genotypes for infantile spasms, including Miller-Dieker, Down and Williams syndromes. Infrequent associations occur with Smith-Lemli-Opitz, Sotos syndrome, and neurofibromatosis type 1. Unknown predisposing genotypes are suspected in some patients with a global developmental disorder and nonspecific cranial imaging malformations. Aicardi syndrome is an example of a developmental disorder of unknown genotype with unifying phenotype and prominent association with infantile spasms. It affects only females, suggesting a gene located on the $\mathrm{X}$ chromosome.

Infantile spasms associated with hypoxic-ischemic encephalopathy or infection may exhibit imaging patterns such as atrophy, calcifications and white matter intensities, suggesting an overlap with predisposing genotypes. Hypoxia or infection could represent a "second hit" in a population made vulnerable to spasms by an undiscovered predisposing genotype. A prospective study of prevalence of infantile spasms in patients with perinatal hypoxia or meningitis is needed to investigate this hypothesis. Children with patterns of extrinsic injury are prime candidates for further study of possible genetic mutations. (Paciorkowski AR, Thio LL, Dobyns WB. Genetic and biological classification of infantile spasms. Pediatr Neurology Dec 2011;45:355-367). (Respond: Dr Paciorkowski, Center for Integrative Brain Research, Seattle Children's Research Institute, 1900 Ninth Ave, MS C95-10, Seattle, WA 98101. E-mail: arpac@u.washington.edu).

COMMENT. The authors propose a primary biologic link between infantile spasms and autism, emphasizing connections between infantile spasms and phenotypes other than intractable epilepsy, including tuberous sclerosis. West's son James, the first patient reported with the syndrome, exhibited symptoms compatible with autism, as described by Langdon-Down (of Down's syndrome) who treated the child in later life. (Pies NJ et al. Brain Dev 2003;25:84-101). Patients with infantile spasms show 